\title{
The Acute Asthma: It's just a Respiratory Disease? A Speckle Tracking Echocardiography Study
}

Ruiz-Bailén $\mathbf{M}^{1^{*}}$, Cobo-Molinos $\mathrm{J}^{1}$, Espada-Fuentes $\mathrm{JC}^{2}$, Castillo-Rivera $\mathrm{AM}^{1}$, Martínez-Ramírez $\mathrm{MJ}^{1}$ and Cárdenas-Cruz $\mathrm{A}^{2}$

${ }^{1}$ Departamento de Ciencias de la Salud, Universidad de Jaén, Spain

${ }^{2}$ Departamento de Medicina, Universidad de Granada, Spain

*Corresponding author: Ruiz-Bailén M, Departamento de Ciencias de la Salud, Universidad de Jaén, Spain, Tel: +34 953 212121 ; Fax: +34 953 212239 ; E-mail: ruizbailen@gmail.com

Received date: October 13, 2016; Accepted date: October 26, 2016; Published date: November 3, 2016

Copyright: ( $) 2016$ Ruiz-Bailén M, et al. This is an open-access article distributed under the terms of the Creative Commons Attribution License, which permits unrestricted use, distribution, and reproduction in any medium, provided the original author and source are credited.

\begin{abstract}
Objective: To determine the presence of acute myocardial dysfunction in status asthmaticus.

Methodology: Prospective studies were included acute severe asthma patients admitted to intensive care from January 2008 to September 2013. We included a control group with healthy athletes subjected to stress by "bench press $n=20$-" and "combat karate for 15 minutes, n=14-", SC2000-2013 $\square$ Siemens Echocardiography was available in the gym. Cardiac stress response of asthmatic patients and athletes was evaluated by echocardiography. An offline analysis by 3D Image and hybrid speckle tracking was performed. We performed analysis by means of using ANOVA test.
\end{abstract}

Results: 32 asthmatic patients (11 males) were included, with a median age of 31 (17-40 years). The age of the controls was $33.45 \pm 0.9$ years. The left ventricular ejection fraction was similar although septum and anterolateral segment presented segmental alterations in all acute asthmatics patients evidenced by strain and strain rate. Acute asthmatics patients had lower strain, lower velocities, less displacements, and more asynchrony versus controls.

Conclusions: Acute asthmatics patients were complicated with a myocardial dysfunction detected by speckle tracking. This tool could be useful for the proper diagnosis of critically ill patients.

Keywords: Status asthmaticus; Myocardial dysfunction; Echocardiography; Stress cardiomyopathy; Stress cardiomyopathy; Strain; Strain rate; Intensive care medicine

Abbreviations: ICU: Intensive Care Unit; S: Strain; SR: Strain Rate; LVEF: Left Ventricular Ejection Fraction; RVEF: Right Ventricular Ejection Fraction; EDLVV: En Diastolic Left Ventricular Volume; VTI: Velocity Time Integral

\section{Introduction}

Status asthmaticus or severe asthma [1] is a common cause of admission to Intensive Care Medicine (ICU) requiring aggressive maneuvers among them are steroids, bronchodilators, methylxanthines, anticholinergic drugs that could have a potentially cardio toxic effect $[2,3]$.

Acute cardiomyopathy is described in critically ill patients, with an unknown etiology, which is attributed to stress, inflammatory condition or possible catecholaminergic consequences. Takotsubo or stress cardiomyopathy occurs in situations of severe stress, with an unknown etiology, but that could change in the survival and could settle on patients with severe non cardiac pathologies. In some patients with status asthmaticus this complication has been detected [4-6].

Our hypothesis is that the development of stress cardiomyopathy may be underdiagnosed in status asthmatic, since it is assumed as a purely respiratory disease. Therefore, it is the unusual electrocardiograms or cardiac examination by echocardiography. Echocardiography is not usually done during status asthmaticus. When this technique is performed on a left ventricular ejection fraction (LVEF) is usually assessed and cannot detect stress cardiomyopathy. Speckle Tracking is a new echocardiographic tool to evaluate the myocardial fibers functionality by

- The systolic velocities and diastolic peaks

- Displacement

- The deformity (strain) of the myocardial fibers, or the rate of deformity (strain rate)

- Evaluation of the degree of synchrony between the different myocardial fibers.

Altering these patterns would suggest varying degrees of myocardial dysfunction. This new tool could detect more episodes of this complication, which could generate new pathophysiological and therapeutic approaches. Our objective is to explore the possible existence of stress cardiomyopathy by speckle tracking echocardiography in severe acute asthma patients requiring admission to ICU.

\section{Methods}

\section{Study design}

Prospective study, Asthmatics group was in which we consider suffering stress at the critical situation, being critically ill patients. Just 
Citation: Ruiz-Bailén M, Cobo-Molinos J, Espada-Fuentes JC, Castillo-Rivera AM, Martínez-Ramírez MJ, et al. (2016) The Acute Asthma: It's just a Respiratory Disease? A Speckle Tracking Echocardiography Study. J Clin Respir Dis Care 2: 122. doi: $10.4172 / 2472-1247.1000122$

Page 2 of 4

another group of healthy people was used to view the values considered normal for our group. Therefore, we use a control group, we subject to stress through exercise, leading to a situation of extreme exhaustion. The case group was composed of:

- status asthmaticus requiring ICU admission without previous comorbidity

- less than 40 years of age, to rule out coronary disease

- absence of family or own history of cardiovascular events, diabetes and chronic hypertension, predisposing to ischemic heart disease

- Possibility of echocardiography in the first 24 hours after admission to ICU

- Accepting inclusion in the study.

The control group consisted of healthy athletes subjected to stress by exercising in gyms by "bench press-n=20 -" and "kumite Shotokan karate" for 15 minutes, $n=14-"$. Echocardiography was available in the gym itself "SC 2000-2013 Siemens囚". Echocardiography was performed immediately before and after bench press or karate combat. For the bench press the following protocol was used. Degree of strength training will be assessed by the maximum load for 1RM. The following protocol was used to evaluate the strength and prevent injuries:

- Start with a warm-up set with a light load allowing you to perform 10 repetitions.

- Rest for one minute.

- Perform another series with a resistance heating that will allow complete 3-5 repetitions. This usually means increased $5-10 \%$ of the previous set.

- Rest for two minutes.

- Estimate another increase (5-10\%) that allowed you to fully 2 or 3 repetitions.

- Rest for four minutes.

- Estimate another increase (5-10\%) that allows for a single repetition of the exercise.

- Break four minutes and then calculate a new moderate increase in weight (5-10\%), and repeat the test.

If you cannot lift the weight, after break of four minutes, the weight (2.5 to 5\%) will be reduced, and then repeat. Continue increasing or decreasing the weight as necessary to determine their repetition maximum (RM) real. After determining the training load using the $1 \mathrm{RM}$ test, the load can move a total of 10 replicates were estimated. Training protocol is based on the 4 sets of 10 repetitions with a load current of $75 \%$ of $1 \mathrm{RM}$ estimating, each with a break of 1'30 "between sets, during the conduct of the exercise, series and rest included, perform echocardiography. Heart stress response of asthmatic patients and athletes were evaluated by echocardiography. An "off-line" analysis was performed by "Velocity Vector Image, Software syngo, Siemens ", U.S. 2013" and "3D Image hybrid speckle tracking".

Study was carried in the Intensive Care Unit of the Hospital Medical Surgical University Hospital of Jaén. The studio on the controls, both the basal and stress was conducted in gym "Emotion Sport Club of Jaén - http://emotionsportsclub.com/-” It belongs to PAIDI CTS 606 Andalusian Health Service Project No. PI-0585-2012, approved by the local ethics committee, and funded by the department of Health of the Government of Andalusia, Government Andalusia, Spain.

\section{Intervention and Clinical Cohort}

All patients were managed following the decision of the responsible intensivist as usual, using different drugs (salbutamol, magnesium, epinephrine, ipratropium bromide, sodium hemisuccinate 6methylprednisolone) and sedation required. In the group of athletes echocardiography was performed in the gym before and after exercise. The stress test in the athlete was performed using a bench press and by a karate fighter.

\section{Image acquisition and processing}

A standard transthoracic echocardiogram was performed with Sequoia 512. We use the probes $3 \mathrm{VC}$ and $4 \mathrm{VC}$. A digital recording studio was done. Echocardiography was performed using acoustic catches a frame rate greater than $60 \mathrm{~Hz}$. We evaluated the usual echocardiographic parameters, quantification of biventricular systolic function (LVEF and RVEF), E/E' ratio, estimating the pulmonary capillary wedge pressure (PCWP) and parameters derived from speckle tracking, such as strain, strain rate, radial displacement, longitudinal and radial velocities on left ventricle. Off line analysis was performed by Syngo software, U.S. Siemens $\ 2013$. LVEF was assessed by the Simpson method. We assessed the global S and SR according to a 16-segment model. The $S$ and SR of the right ventricle were assessed in apical 4 chambers, including the lateral and septal side. We consider as strain and longitudinal strain rate, when evaluated in the apical projection 4 chambers corresponding to the longitudinal fibers. The synchrony was evaluated by measuring the delay of the systolic velocity peaks between the septal and lateral walls by Speckle Tracking.

\section{Statistical analysis}

A study for quantitative variables was performed using ANOVA. We have used Bland-Altman analyses for intra-observer variability. Their results are presented using means and standard deviations. It was regarded as $p$ value $<0.05$ as statistically significant.

\section{Results}

Thirty-two asthmatic patients (11 males) were included, with a median age of 31 (17-40 years). All required mechanical ventilation for a median of 3 days range ( 0.3 to 16 ). The survival rate was $100 \%$. The age of the controls was $33.45 \pm 0.9$ years. None electrocardiographic changes occurred in athletes. During the first 12 hours there was STsegment elevation in left precordial in 9 patients with asthma who were normalized in the first 5 days, there was also flattening of the $\mathrm{T}$ wave from V1 to V3 in 12 other asthmatics. The mean value of troponin I was $0.12 \mathrm{pg} / \mathrm{dl}$, with a peak of $6.33 \mathrm{pg} / \mathrm{dl}$. The E/E' ratio and the PCWP estimated by equation Nagueh were much higher in asthmatics patients, which showed a clear elevation of these pressures, at least suggestive of diastolic dysfunction, and possible occurrence of cardiogenic edema. The LVEF was similar, although in all asthmatics patients strain, strain rate and longitudinal velocity in septum and anterolateral segments were decreased. Twelve asthmatic patients had apical ballooning by 2D echocardiography. The left ventricular dyssynchrony, assessed by the delay of the average longitudinal velocity between the septal and lateral segments was higher in status asthmaticus. Acute asthmatics patients had finally lower:

- Strain

- Velocities, displacements, and more asynchrony; versus controls. 
Citation: Ruiz-Bailén M, Cobo-Molinos J, Espada-Fuentes JC, Castillo-Rivera AM, Martínez-Ramírez MJ, et al. (2016) The Acute Asthma: It's just a Respiratory Disease? A Speckle Tracking Echocardiography Study. J Clin Respir Dis Care 2: 122. doi: $10.4172 / 2472-1247.1000122$

Page 3 of 4

- Unfortunately, only 19 patients were followed for one month, and all their echocardiographic values were normalized, although their mean global S was (-16).

There was no intraobserver variability for the $S$ and SR. Intraobserver variability in strain on these patients was good with correlation coefficient 0.86 for strain, 0.82 for strain rate), and 0.731 for ejection fraction (Table 1) (Figures 1 and 2).

\begin{tabular}{|c|c|c|c|c|}
\hline Variable & Asthma status & Basal control & Stress control & $\mathbf{P}$ \\
\hline Heart rate & $127.37 \pm 21.67$ & $62.01 \pm 21.35$ & $112.99 \pm 19.47$ & 0.0001 \\
\hline LVEF & $0.49 \pm 0.11$ & $0.56 \pm 0.08$ & $0.63 \pm 0.08$ & 0.056 \\
\hline Left Longitudinal S & $-14.45 \pm(-0.36)$ & $-20.24 \pm(-5.44)$ & $-26.83 \pm(-4.24)$ & $<0.0001$ \\
\hline Left longitudinal SR & $-1.04 \pm(-0.47)$ & $-1.55 \pm(-0.35)$ & $-2.36 \pm(-0.55)$ & $<0.001$ \\
\hline Left PCwP estimated by Nagueh & $14.23 \pm 0.18$ & $4.88 \pm 0.03$ & $6.09 \pm 0.23$ & $<0.01$ \\
\hline Left septal velocity wave "s" (m/s) & $0.089 \pm 0.16$ & $0.104 \pm 0.07$ & $0.181 \pm 0.09$ & $<0.0001$ \\
\hline Average longitudinal velocity LV $(\mathrm{cm} / \mathrm{s})$ & $2.21 \pm 0.09$ & $4.43 \pm 1.29$ & $6.13 \pm 1.37$ & $<0.0001$ \\
\hline Left time delay longitudinal velocity (ms) synchrony & $162.38 \pm 0.47$ & $106.12 \pm 0.48$ & $88.78 \pm 0.68$ & $<0.0001$ \\
\hline Average radial velocity $\mathrm{LV}(\mathrm{cm} / \mathrm{s})$ & $1.87 \pm 0.13$ & $2.86 \pm 0.97$ & $4.68 \pm 1.61$ & $<0.0001$ \\
\hline Left longitudinal displacement $(\mathrm{mm})$ & $4.53 \pm 0.27$ & $7.57 \pm 2.43$ & $8.87 \pm 1.75$ & $<0.01$ \\
\hline LV Tei index & $0.68 \pm 0.16$ & $0.38 \pm 0.04$ & $0.33 \pm 0.006$ & 0.055 \\
\hline Right IVA $\left(\mathrm{m} / \mathrm{s}^{2}\right)$ & $0.98 \pm 0.01$ & $1.36 \pm 0.18$ & $3.21 \pm .47$ & 0.031 \\
\hline Right Longitudinal S & $-14.36 \pm(-0.88)$ & $-25.78 \pm(-3.11)$ & $-33.17 \pm(-3.18)$ & 0.065 \\
\hline Right longitudinal SR & $-0.94 \pm(-0.91)$ & $-1.58 \pm(-0.47)$ & $-2.55 \pm(-0.91)$ & $<0.001$ \\
\hline Right area of fractional shortening & $0.42 \pm 0.11$ & $0.45 \pm 0.15$ & $0.49 \pm 0.033$ & 0.182 \\
\hline TAPSE (mm) & $16.86 \pm 1.34$ & $17.88 \pm 1.23$ & $21.24 \pm 2.49$ & 0.452 \\
\hline Right lateral velocity wave "s" (m/s) & $0.099 \pm 0.21$ & $0.091 \pm 0.08$ & $0.19 \pm 0.013$ & 0.09 \\
\hline
\end{tabular}

Table 1: Echocardiographic values of three groups.

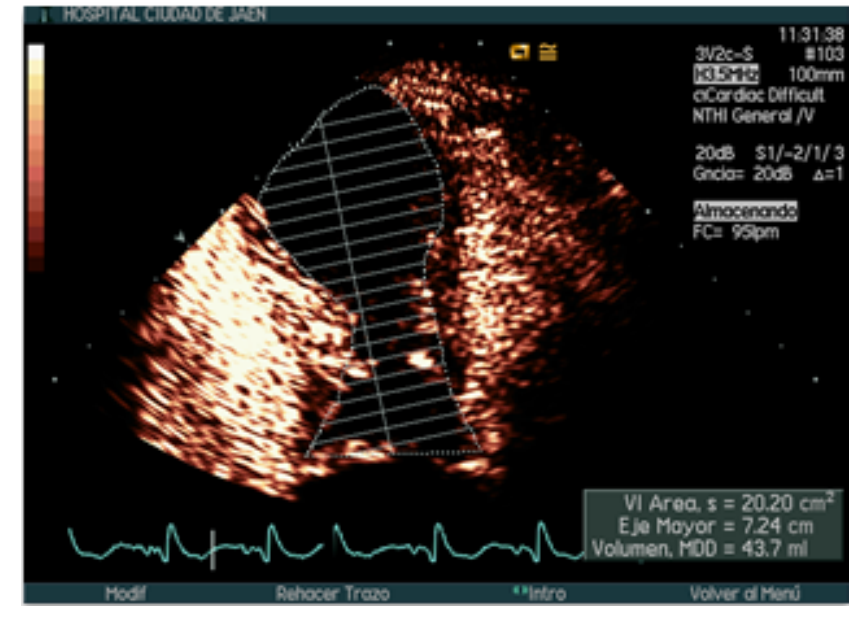

Figure 1: Morphology of the left ventricle of a patient with acute asthma complicated with heart failure.

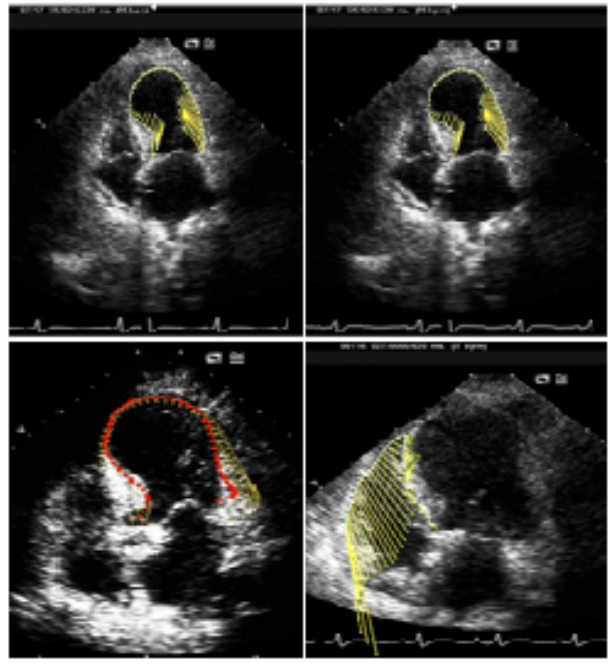

Figure 2: Speckle tracking image. 
Citation: Ruiz-Bailén M, Cobo-Molinos J, Espada-Fuentes JC, Castillo-Rivera AM, Martínez-Ramírez MJ, et al. (2016) The Acute Asthma: It's just a Respiratory Disease? A Speckle Tracking Echocardiography Study. J Clin Respir Dis Care 2: 122. doi: 10.4172/2472-1247.1000122

Page 4 of 4

\section{Discussion}

Status asthmaticus is a critical situation of difficult control [7]. The etiology of asthmatic condition may be multifactorial, but usually are patients with a possible inflammatory state and regularly requires high doses of cardiotoxic drugs. It has already been reported [6], the asthmatic condition can cause stress cardiomyopathy. Despite the obvious limitations of our study, it could suggest the hypothesis that asthmatic patients may have alterations in echocardiographic parameters and elevated diastolic pressures. Interestingly the use of speckle tracking has been a very useful tool for the detection of myocardial dysfunction because this might have gone unnoticed if we had evaluated only the LVEF. The control group was probably not necessary but we decided to include them to see the differences observed with stress among asthma patients and athletes. Even understanding that management goes through a strict treatment could be to decrease the resistance during handling can be interesting to perform an echocardiogram to evaluate at least the $\mathrm{E} / \mathrm{E}^{\prime}$, a fact that could certainly make us change our therapeutic response. In the estimation of high diastolic pressures, we should consider vasodilators and decrease the cardiotoxic therapy.

There is difficulty in explaining the cardiotoxicity, whether due to the inflammatory state induced asthma, the underlying disease, the use of mechanical ventilation or a large dose of bronchodilator treatment or excess catecholamine's (which could cause catecholaminergic cardiomyopathy), or by cardiotoxicity drugs. All present could contribute to a deleterious effect, and even generate a cardiogenic shock that might go unnoticed or even mistaken for a coronary syndrome. Echocardiography at the bedside is very necessary for the proper therapeutic approach. And new tools such as strain, strain rate, should be incorporated into the diagnostic arsenal intensivists. The speckle tracking can alert complication before the development of segmental contractility disorders, thanks to its high sensitivity. This could change the management.

Another important aspect is the existence of right dysfunction, which could be explained by asthma itself, by the changes in transmural pressures or even by the stress cardiomyopathy.
The results of this study should be interpreted with caution, since speckle tracking is not used in intensive care. In addition this diagnostic tool can be modified slightly by the load.

\section{Conclusions}

Despite normal global LVEF during the status asthmatic myocardial dysfunction by speckle tracking is detected. This tool is useful for the proper diagnosis of the critical patient.

\section{Funding}

Servicio Andaluz de Salud. SAS Project No. PI-0585-2012. Approved by the local ethics committee, and funded by the "Consejería de Salud" of the Government of Andalusia. PAIDI CTS-606. Andalusian Government. Spain.

\section{References}

1. Schivo M, Phan C, Louie S, Harper RW (2013) Critical asthma syndrome in the ICU. Clin Rev Allergy Immunol 48: 31-44.

2. Yock Corrales A, Soto-Martínez M, Starr M (2011) Management of severe asthma in children. Aust Fam Physician 40: 35-38.

3. Gupta P, O'Mahony MS (2008) Potential adverse effects of bronchodilators in the treatment of airways obstruction in older people: recommendations for prescribing. Drugs Aging 25: 415-443.

4. Romero-Bermejo FJ, Ruiz-Bailén M, Gil-Cebrian J, Huertos-Ranchal MJ (2011) Sepsis-induced cardiomyopathy. Curr Cardiol Rev 7: 163-183.

5. Castillo RAM, Ruiz-Bailén M, Rucabado Aguilar L (2011) Takotsubo cardiomyopathy--a clinical review. Med Sci Monit 17: RA135-RA147.

6. Ruiz-Bailén M, Aguayo de Hoyos E, Ruiz-Navarro S, Díaz-Castellanos MA, Rucabado-AL, et al. (2005) Reversible myocardial dysfunction after cardiopulmonary resuscitation. Resuscitation 66: 175-181.

7. Peters JI, Stupka JE, Singh H, Rossrucker J, Angel LF, et al. (2012) Status asthmaticus in the medical intensive care unit: a 30-year experience. Respir Med 106: 344-348. 